\title{
Multistable alignment states in nematic liquid crystal filled wells
}

\author{
C. Tsakonas \\ School of Biomedical and Natural Sciences, The Nottingham Trent University, Clifton Lane, Nottingham \\ NG11 8NS, United Kingdom
}

\author{
A. J. Davidson \\ Department of Mathematics, University of Strathclyde, Glasgow, G1 1XH Scotland, United Kingdom \\ C. V. Brown \\ School of Biomedical and Natural Sciences, The Nottingham Trent University, Clifton Lane, Nottingham \\ NG11 8NS, United Kingdom
}

\author{
N. J. Mottram ${ }^{\text {a) }}$ \\ Department of Mathematics, University of Strathclyde, Glasgow, G1 1XH Scotland, United Kingdom
}

(Received 15 December 2006; accepted 3 February 2007; published online 16 March 2007)

\begin{abstract}
Two distinct, stable alignment states have been observed for a nematic liquid crystal confined in a layer with thickness of $12 \mu \mathrm{m}$ and in square wells with sides of length between 20 and $80 \mu \mathrm{m}$. The director lies in the plane of the layer and line defects occur in two corners of the squares. The positions of the defects determine whether the director orientation is across the diagonal or is parallel to two opposite edges of the square. The device is multistable because both the diagonal and parallel states are stable when rotated by multiples of $90^{\circ}$ in plane. (C) 2007 American Institute of Physics. [DOI: 10.1063/1.2713140]
\end{abstract}

Liquid crystal displays are now a ubiquitous flat panel and portable display technology. A unifying feature in these devices is the requirement for an active matrix backplane to continuously apply voltage wave forms at every pixel. A number of liquid crystal display modes have been developed that exhibit bistability, which possess two zero voltage minimum energy orientations. Such modes can be driven using multiplex addressing on a simple passive matrix. Azimuthal bistability, where the two stable states occur at different inplane rotation angles in the liquid crystal layer, has been achieved using surface gratings, surface bigratings, and periodic arrays of post structures. ${ }^{1-3}$ Zenithal bistability, where the two stable director configurations differ in their net outof-plane tilt, has been achieved in devices using surface grating structures. ${ }^{1,4,5}$ A silicon oxide film can also give rise to two equivalent easy directions of a nematic liquid crystal at different tilt and azimuthal angles. ${ }^{6,7}$ Examples of bistable devices where one state is untwisted but the other state has a nematic director orientation that twists through the thickness of the liquid crystal layer are described in Refs. $8\left(0^{\circ}\right.$ and $\left.360^{\circ}\right)$ and $9\left(0^{\circ}\right.$ and $\left.180^{\circ}\right)$. Cholesteric liquid crystals, which have tight helical pitch values, have long been known to give two stable textures (planar and focal conic) when combined in a thin layer and this effect has more recently been exploited for display applications. 10,11

In the current work azimuthal bistability, and multistability, has been achieved by the confinement of nematic liquid crystal in a square well. ${ }^{12}$ A schematic diagram showing a section through the device is shown in Fig. 1. The photoresist that is used to define the well structure imparts random planar anchoring to the nematic liquid crystal. This means that the easy direction of the nematic director at the surface of the wells is parallel to the surface but there is no preferred direction parallel to the well wall. The height of the well, corresponding to the thickness of the liquid crystal layer, is

\footnotetext{
${ }^{a)}$ Electronic mail: nigel.mottram@strath.ac.uk
}

kept below half the dimension of the length of the sides of the well. With this aspect ratio and the random planar surface anchoring conditions, the nematic liquid crystal director lies in the plane of the layer as shown in Fig. 1.

The substrates of the device were borosilicate glass slides with thickness of $1.1 \mathrm{~mm}$ that had been precoated with indium tin oxide with sheet resistance of $100 \Omega$ /sq. SU8 photoresist was used for the dielectric layers and wall structures within the device. SU8 is a negative resist that has been developed for applications requiring surface structures with smooth well-defined walls and thick layers. ${ }^{13}$ It is suited for this application where permanent structures made from the cured material are in contact with liquid crystal material because cured SU8 is resistant to solvents and is thermally stable. The dielectric surface layers were formed from a $2 \mu \mathrm{m}$ layer of SU8 photoresist deposited directly onto each indium tin oxide (ITO)/glass substrate. Arrays of wells were fabricated from SU8 using standard photolithography onto one of the glass/ITO substrates that had been coated with a $2 \mu \mathrm{m}$ layer of SU8. The devices consisted of one substrate with the dielectric layer and the well pattern and one substrate with only the dielectric layer, as shown in Fig. 1. Before assembly the commercial liquid crystal E7 (Ref. 14) was filled in the isotropic phase into the wells by a doctor-blade technique. After assembly the substrates were held together by pressure and bonded using UV adhesive NAO65. ${ }^{15}$

Upon cooling from well above the isotropic-nematic phase transition, optical textures of the nematic-filled wells

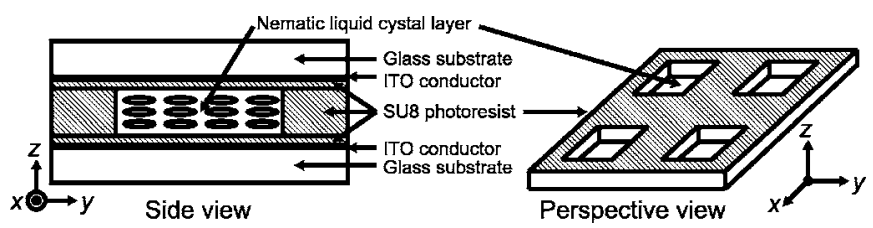

FIG. 1. Schematic diagram of the nematic liquid crystal device. The nematic liquid crystal is confined by a periodic series of wells in the $x-y$ plane. 


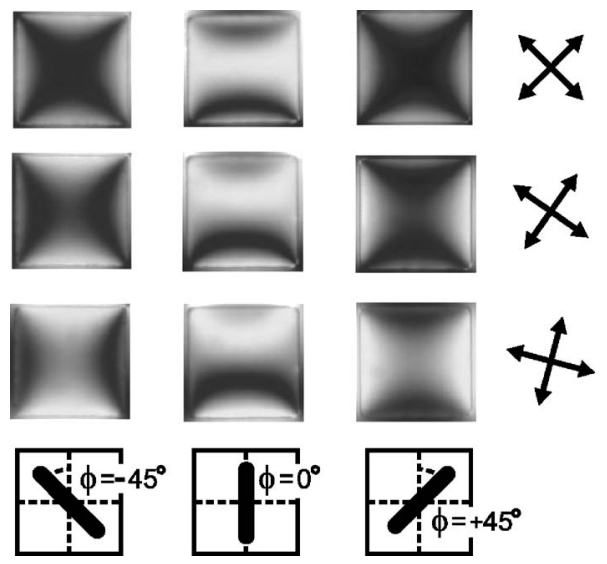

FIG. 2. Alignment states observed in transmission between crossed polarizers under a magnification of $100 \times$. The polarizers are rotated by $0^{\circ}$ (upper row), $10.5^{\circ}$, and $21.0^{\circ}$ (lower row) relative to the device. The arrows in each case indicate the polarizer orientations. The wells were formed from squares with side of $80 \mu \mathrm{m}$.

were observed between crossed polarizers at a magnification of $100 \times$. Of the different types of optical texture that occurred in wells with side of $80 \mu \mathrm{m}$, three are shown in the three columns in Fig. 2. Each row shows the appearance of a particular texture for a different orientation of the polarizers, indicated by the arrows to the right of each row.

In the top row of Fig. 2, the left hand and right hand wells exhibit textures with two axes of symmetry, whereas the well shown in the center exhibits an optical texture that is symmetrical about a single axis, the vertical center line. The transmission between crossed polarizers of in-plane nematic optical texture exhibits a minimum in the regions where the nematic director is parallel to either of the polarizers. ${ }^{16}$ The right and left hand textures therefore contain large regions where the nematic director is either parallel or perpendicular to the diagonal since the polarizers are aligned along the diagonals and large regions of the textures appear dark. Conversely, the large light area in the central texture indicates regions where the director is substantially parallel to the edges of the square well. For all three textures, the light regions close to all the sides of the square edges are consistent with tangential alignment there due to the random planar alignment at the SU8 surface.

As the polarizers are rotated clockwise, the cross shapes of the dark regions of the left and right hand wells develop lighter regions in the center of the square with separated dark brushes moving until they are along the opposite sides of the squares in the bottom row. In the left hand well these brushes are parallel to the vertical edges of the square, whereas in the right hand well they are parallel to the horizontal edges. This indicates that for the left hand well the nematic director is substantially parallel to the diagonal running between the top left hand and the lower right hand corner. This will be referred to as a $\phi=-45^{\circ}$ in-plane rotation state. For the right hand well the director at the center is oriented substantially along the orthogonal diagonal. This will be referred to as a $\phi=+45^{\circ}$ in-plane rotation state. The texture in the central well has the nematic director substantially vertical and so this will be referred to as a $\phi=0^{\circ}$ in-plane rotation state. These orientations are indicated at the bottom of Fig. 2.

The assignments for the director orientations in the centers of the wells shown in Fig. 2 have been confirmed by numerical modelling. Figure 3 shows the results of simulaDownloaded 09 Nov 2009 to 152.71.195.24. Redistribution subject

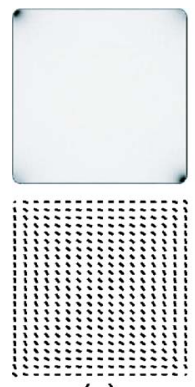

(a)

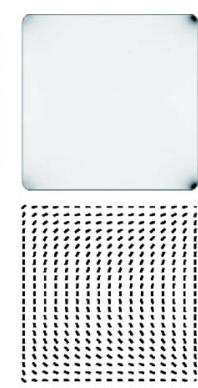

(b)

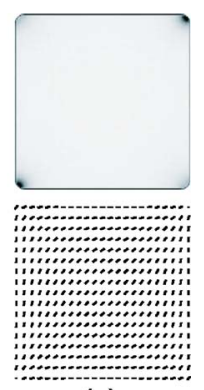

(c)
FIG. 3. (Color online) Alignment states, predicted by $Q$-tensor theory, which correspond to the same states observed in the experimental device in Fig. 2. The upper row shows the two-dimensional variation of the order parameter $S$ as a grayscale plot where white corresponds to $S=0.6$ and black to $S=0.0$. The lower row shows the two-dimensional variation of the inplane rotation angle of the nematic director.

tions of the nematic director orientation in this geometry using the Landau-de Gennes $Q$-tensor theory in two dimensions. ${ }^{17,18}$ This theory has been applied in the literature to nematic and twisted nematic liquid crystal displays and phase grating structures ${ }^{19-21}$ and involves the minimization of the free energy of the nematic layer,

$$
\begin{aligned}
F= & \int_{V}\left[\frac{K}{4 S^{2}}(\nabla \mathbf{Q})^{2}+a \operatorname{tr}\left(\mathbf{Q}^{2}\right)+\frac{2 b}{3} \operatorname{tr}\left(\mathbf{Q}^{3}\right)+\frac{c}{2} \operatorname{tr}\left(\mathbf{Q}^{2}\right)^{2}\right] d \nu \\
& +\int_{S} \frac{3 W}{2 S^{2}} \boldsymbol{\nu} \cdot \mathbf{Q} \cdot \boldsymbol{\nu} d s
\end{aligned}
$$

where tr denotes the trace of a matrix and $\boldsymbol{\nu}$ is the normal vector at the boundary of the liquid crystal region. The first term in Eq. (1) is due to elastic distortions, the next three terms are the Landau-de Gennes expansion and govern the thermotropic equilibrium state of the order parameter, and the last energy term is a surface energy which characterises the planar degenerate anchoring at the walls of the well. For the current work, values for the coefficients of the powers in the Landau-de Gennes energy $\left(a=-0.112 \times 10^{6} \mathrm{~N} \mathrm{~m}^{-2} \mathrm{~K}^{-1}\right.$, $b=-0.64 \times 10^{6} \mathrm{~N} \mathrm{~m}^{-2}$, and $c=1 \times 10^{6} \mathrm{~N} \mathrm{~m}^{-2}$ ) were taken to be of the same order as those in Ref. 22 where the specific numerical values were chosen to give a bulk order parameter of $S=0.6$, a typical value. Strong degenerate planar anchoring was assumed at the surfaces ${ }^{23}$ with an equivalent anchoring coefficient of $W=2 \times 10^{-3} \mathrm{~N} \mathrm{~m}^{-1}$ and for the elastic energy term an equal elastic constant approximation, $K=1$ $\times 10^{-11} \mathrm{~N}$, was used.

Figure 3 shows the calculated two-dimensional variation of the order parameter $S$ (top row) and the nematic director for three different alignment textures for squares with side of $1 \mu \mathrm{m}$ (bottom row). A scaleup of the $x-y$ dimensions to match the experimental setup is possible but increases the computational demand and will only be important if dynamical switching is modeled. The magnitude of $S$ is indicated by grayscale, with white corresponding to $S=0.6$ and black corresponding to $S=0.0$. The dark spots in the corners of the squares indicate the positions of defects. The sizes of the defects are consistent with the nematic coherence length of around $50 \mathrm{~nm} .{ }^{17}$ In Fig. 3(a), the director lies along the diagonal in the center of the well in the direction $\phi=-45^{\circ}$. This state arises when the defects are in the upper left and lower right hand corners. In Fig. 3(c), the director lies along the orthogonal diagonal, $\phi=+45^{\circ}$, and the defects occur in to AIP license or copyright; see http://apl.aip.org/apl/copyright.jsp 


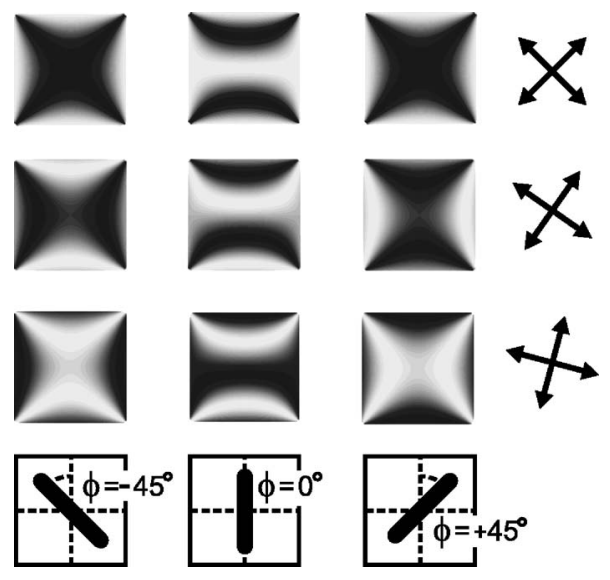

FIG. 4. Optical textures produced from the modeled alignment states shown in Fig. 3 assuming that the transmission between crossed polarizers is given by the standard formula for a birefringent material (Ref. 24). The alignment states are shown with polarisers rotated by $0^{\circ}$ (upper row), $10.5^{\circ}$, and $21.0^{\circ}$ (lower row) relative to the device.

the opposite corners. In contrast, the director is vertical in the central region of the square, $\phi=0^{\circ}$, in Fig. 3(b). For this director configuration the defects now occur in the two corners on the right hand edge of the square.

In Fig. 4, the predicted optical textures between crossed polarizers are shown for the same orientations of crossed polarizers that were used in Fig. 2 when observing the alignment textures that are observed in an experimental device. The theoretical optical textures were generated, at each point in the plane, from the equation for the fraction of incident light intensity transmitted $T$ by a uniaxial birefringent material between crossed polarizers, $T=\sin ^{2}(2 \phi) \sin ^{2}(\pi d \Delta n / \lambda){ }^{24}$ The maximum transmission depends on the thickness $d$ of the layer, the birefringence $\Delta n$, and the wavelength $\lambda$ of the incident light. The similarity between the theoretically generated optical textures in Fig. 4 and the textures observed in an experimental device in Fig. 2 is remarkable and this confirms the assignments of $\phi=-45^{\circ}, \phi=0^{\circ}$, and $\phi=+45^{\circ}$ for the in-plane rotation angle in the centers of the wells that have been given to the three different states.

The diagonal alignment texture has been shown to occur in either of two possible orthogonal orientations, $\phi=-45^{\circ}$ and $\phi=+45^{\circ}$. The two-dimensional director distortion profiles associated with these states, shown in Fig. 3, are identical except for a rotation of $90^{\circ}$. Therefore these states would be expected to be energetically degenerate. For the vertical alignment texture, $\phi=0^{\circ}$, exactly the same twodimensional director distortion profile if the texture is rotated by multiples of $90^{\circ}$. For this alignment state there are therefore four energetically degenerate states corresponding to inplane rotations of $0^{\circ}, 90^{\circ}, 180^{\circ}$, and $270^{\circ}$. The square well geometry is therefore capable of producing a total of six different two-dimensional director alignment configurations.
To summarize, multistable alignment of nematic liquid crystal confined in square wells has been demonstrated both theoretically and in experimental devices. $Q$-tensor modeling predicts two distinct alignment geometries (up to rotations), one where nematic defects occur in opposite corners of the square well and one where defects occur in two corners on the same side of the square well. Optical textures observed by polarizing microscopy in experimental devices can be very well reproduced using the theory. The device structure is relatively easy to fabricate because the random planar alignment that naturally occurs at an untreated photoresist surface is exploited to produce alignment of the nematic director in the plane of the substrates and no additional surfactant treatment is required.

The authors gratefully acknowledge Scottish Enterprise and the Engineering and Physical Sciences Research Council for funding.

${ }^{1}$ R. N. Thurston, J. Cheng, and G. D. Boyd, IEEE Trans. Electron Devices ED27, 2069 (1980).

${ }^{2}$ R. Barberi, J. J. Bovent, M. Giocondo, M. Iovane, and A. L. AlexeIonescu, J. Appl. Phys. 84, 1321 (1998).

${ }^{3}$ S. Kitson and A. Geisow, Appl. Phys. Lett. 80, 3635 (2002).

${ }^{4}$ G. P. Bryan-Brown, C. V. Brown, and J. C. Jones, U.K. Patent No. GB2318422 (16 October 1995).

${ }^{5}$ C. V. Brown, M. J. Towler, V. C. Hui, and G. P. Bryan-Brown, Liq. Cryst. 27, 233 (2000).

${ }^{6}$ M. Monkade, M. Boix, and G. Durand, Europhys. Lett. 5, 697 (1988).

${ }^{7}$ B. Jerome, P. Pieranski, and M. Boix, Europhys. Lett. 5, 693 (1988).

${ }^{8}$ D. W. Berreman and W. R. Heffner, J. Appl. Phys. 52, 3032 (1981).

${ }^{9}$ I. Dozov, M. Nobili, and G. Durand, Appl. Phys. Lett. 70, 1179 (1997).

${ }^{10}$ W. Greubel, U. Wolff, and H. Kruger, Mol. Cryst. Liq. Cryst. 24, 103 (1973).

${ }^{11}$ D.-K. Yang, J. W. Doane, Z. Yann, and J. Glasser, Appl. Phys. Lett. 64, 1905 (1994).

${ }^{12}$ N. J. Mottram, A. Ramage, G. Kelly, and A. J. Davidson, U.K. Patent No. GB20040026582 (8 June 2006).

${ }^{13}$ SU8 (formulated in GBL) chemically amplified epoxy based negative resist. MicroChem Corporation, 1254 Chestnut Street, Newton, MA 02464, USA.

${ }^{14}$ E7 room temperature nematic liquid crystal, Liquid Crystals Division, Merck KGaA, 64271 Darmstadt, Germany.

${ }^{15}$ Norland Optical Adhesive 65, Norland Products, Inc., 2540 Route 130, Suite 100, Cranbury, NJ 08512, USA.

${ }^{16} \mathrm{~S}$. Chandrasekhar, Liquid Crystals, 2nd ed. (Cambridge University Press, Cambridge, 1992), Chap. 3, p. 117.

${ }^{17}$ P. G. de Gennes and J. Prost, The Physics of Liquid Crystals, 2nd ed. (Clarendon, Oxford, 1993), Chap. 2, p. 76.

${ }^{18}$ D. W. Berreman and S. Meiboom, Phys. Rev. A 30, 1955 (1984).

${ }^{19}$ A. Kilian and S. Hess, Z. Naturforsch., A: Phys. Sci. 44, 693 (1989).

${ }^{20}$ S. Dickmann, J. Eschler, O. Cossalter, and D. A. Mlynski, SID Int. Symp. Digest Tech. Papers 1993, 638.

${ }^{21}$ H. Mori, E. C. Gartland, J. R. Kelly, and P. J. Bos, Jpn. J. Appl. Phys., Part 1 38, 135 (1999).

${ }^{22}$ E. B. Priestley, P. J. Wojtowicz, and P. Sheng, Introduction to Liquid Crystals (Plenum, New York, 1974), Chap. 10, p. 168.

${ }^{23}$ M. A. Osipov and S. Hess, J. Chem. Phys. 99, 4181 (1993).

${ }^{24}$ W. A. Crossland and T. D. Wilkinson, in Handbook of Liquid Crystals (Wiley-VCH, Weinheim, 2005), Vol. 1, Chap. IX, p. 771. 